\title{
Functional Conservation of Multiple Elements in Yeast Chromosomal Replicators
}

\author{
HAI RAO, YORK MARAHRENS, $\dagger$ AND BRUCE STILLMAN* \\ Cold Spring Harbor Laboratory, Cold Spring Harbor, New York 11724, and Graduate Program in Molecular and \\ Cellular Biology, State University of New York at Stony Brook, Stony Brook, New York 11794
}

Received 13 May 1994/Returned for modification 17 June 1994/Accepted 8 July 1994

\begin{abstract}
Replicators that control the initiation of DNA replication in the chromosomes of Saccharomyces cerevisiae retain their function when cloned into plasmids, where they are commonly referred to as autonomously replicating sequences (ARSs). Previous studies of the structure of $A R S 1$ in both plasmid and chromosome contexts have shown that it contains one essential DNA element, $A$, that includes a match to the ARS consensus sequence (ACS), and three additional elements, B1, B2, and B3, that are also important for ARS function. Elements $A$ and $B 3$ are bound by a candidate initiator protein called the origin recognition complex and ARS-binding factor 1, respectively. Although the A and B3 elements have been found in other ARSs, sequence comparisons among ARSs have failed to identify B1- and B2-like elements. To assess the generality of the modular nature of yeast replicators, linker substitution mutagenesis of another yeast chromosomal replicator, $A R S 307$, was performed. Three DNA sequence elements were identified in $A R S 307$, and they were demonstrated to be functionally equivalent to the $A, B 1$, and $B 2$ elements present in $A R S 1$. Despite the lack of DNA sequence similarity, the B1 and B2 elements at each ARS were functionally conserved. Single-base substitutions in the core of the $A R S 1 \mathrm{~B} 1$ and $\mathrm{B} 2$ elements identified critical nucleotides required for the function of the $\mathrm{B} 1$ element. In contrast, no single-point mutations were found to affect $B 2$ function. The results suggest that multiple DNA sequence elements might be a general and conserved feature of replicator sequences in $S$. cerevisiae.
\end{abstract}

Chromosomal DNA replication occurs once during each $\mathrm{S}$ phase of the cell cycle. The process is mainly, but not exclusively, controlled at the initiation step following commitment of the cell to a new round of cell division. Studies of Escherichia coli and of both prokaryotic and eukaryotic viruses have revealed that DNA replication initiates at specific sites called replication origins and is controlled by genetic elements called replicators $(22,39)$. In bacteria and viruses, replicators usually consist of multiple short DNA sequences. Proteins recognize sequences within the replicator, leading to assembly of an initiation complex that catalyzes the initiation reaction, and in some cases the mechanism of initiation has been deduced (24). The central protein of the initiation reaction is referred to as the initiator; however, several additional proteins are always required.

In eukaryotes, in contrast, the mechanism of initiation at cellular origins of DNA replication is poorly defined, although studies are most advanced in the yeast Saccharomyces cerevisiae. Autonomously replicating sequences (ARSs) were identified in $S$. cerevisiae by the ability of chromosome-derived DNA sequences to allow plasmids to be stably maintained (19, 41) and have subsequently been shown to direct the initiation of DNA replication at an origin located close to or within the ARSs $(5,17,20,21)$. Origins of DNA replication in the chromosome have been mapped by two-dimensional agarose gel electrophoresis techniques, and they colocalize to a subset of ARSs $(5,13,17,21)$. Mutations in ARS sequences that abolish origin activity in plasmids also abolish the origin activity in the chromosome $(10,20,28,36)$.

Mutational analyses of $A R S 1$ revealed that this replicator

\footnotetext{
* Corresponding author. Mailing address: Cold Spring Harbor Laboratory, P. O. Box 100, One Bungtown Rd., Cold Spring Harbor, NY 11724. Phone: (516) 367-8383. Fax: (516) 367-8879.

$\dagger$ Present address: Whitehead Institute and Department of Biology, Massachusetts Institute of Technology, Cambridge, MA 02142.
}

consists of a modular arrangement of at least four short sequence elements (A, B1, B2, and B3) that function both in plasmids and in the chromosome $(27,28)$. The best-understood element is element $\mathrm{A}$, which contains a highly conserved sequence called the ARS consensus sequence (ACS), $5^{\prime}-(\mathrm{A} / \mathrm{T})$ TTTAT(A/G)TTT(A/T)-3' $(6,8,37)$. Extensive mutational analyses have demonstrated that every ARS contains one close match to this consensus and that it is essential for ARS function. Sequences immediately flanking the ACS also affect ARS function, and along with the ACS, they form the essential A element $(4,8,9,20,27,28,36,37,40,44)$. By analogy to bacterial and virus replication systems, element $A$ is likely to be the initiator protein-binding site. A multisubunit origin recognition protein complex (ORC) that specifically recognizes this element in vitro and in vivo has been identified $(3,11)$.

Also clearly defined is element $\mathrm{B} 3$, the binding site for a transcription factor, $\mathrm{ABF} 1(7,12,14,27,35)$. B3 can be replaced by the binding sites for other transcription factors without affecting ARS activity on the plasmid (27). Furthermore, an ABF1-binding site has been identified in a number of other ARSs such as $A R S 2, A R S 120$, and $A R S 121(8,37)$ and can function in a position- and orientation-independent manner $(45,46)$.

The region between elements A and B3 is less conserved but equally important for ARS function $(7,12,14,27,35)$. This region is usually around 70 to $80 \mathrm{bp}$ in size and tends to be AT rich $(8,37)$. Progressive deletions of this region have been carried out with some ARSs, but these studies failed to identify any specific DNA elements in this region of domain $B(4,9$, 33). As a consequence, it variously has been proposed that this region is an easily unwound region that is AT rich with no sequence specificity, a DNA-unwinding element with specific (but poorly understood) structural requirements, or a nuclear matrix attachment site $(1,15,20,31,32,37,43)$. An extensive linker scan analysis in $A R S 1$ identified two short sequence elements (B1 and B2) in this region that have been shown to be 
important both in plasmids and in the chromosome $(27,28)$. However, B1- and B2-like sequences have not been identified at other replicators either by sequence comparisons or by mutational analyses. Walker et al. (46) have suggested that yeast replicators share in common the A and B3 elements and an additional AT-rich sequence ( $72 \mathrm{bp})$. The AT-rich sequence from $A R S 121$ can stimulate replicator activity of the $A R S 1$ and $A R S 307$ essential core sequences; however, B1- and B2-like sequences were not identified in $A R S 121$. Therefore, whether B1 and B2 were conserved in other ARSs remained unclear.

Herein, we begin to assess the generality of the modular organization of replicators by performing a mutational analysis on another chromosomal replicator, $A R S 307$ (previously called C2G1) $(10,17,33,34,44)$. Linker mutations in ARS307 identified a modular array of three short sequence elements that correspond to the $\mathrm{A}, \mathrm{B} 1$, and $\mathrm{B} 2$ elements of $A R S 1$. The DNA sequences in the B1 and B2 elements show little similarity between the $A R S 1$ and $A R S 307$ replicators; however, exchanging the elements between the two ARSs clearly demonstrated that they are functionally conserved. To determine if there are any specific sequence requirements in the B1 and B2 elements, extensive point mutations in the core regions of either $\mathrm{B} 1$ or $\mathrm{B} 2$ from $A R S 1$ were also made. These studies revealed some important base pairs within the $B 1$ element but failed to identify any specific single nucleotides within the B2 region.

\section{MATERIALS AND METHODS}

Yeast and bacterial strains. $E$. coli DH5 $\alpha$ (38) was used for bacterial transformations. E. coli BW313 (dut ung thi-1 relA spoT1 $\mathrm{F}^{\prime} \quad l y s A$ ) was used to generate a uracil-containing template for standard oligonucleotide mutagenesis (25). $S$. cerevisiae host strain SP1 (MATa ura3-52 his3 trp1-289 leu22,113 ade8 can 1 ) was used for yeast transformations (27).

Reagents and media. $E$. coli transformants were grown in Luria broth supplemented with $50 \mu \mathrm{g}$ of ampicillin per $\mathrm{ml}$. Enzymes were obtained from New England Biolabs, Inc. (Beverly, Mass). The Sanger dideoxy method was used for DNA sequencing, and Sequenase enzyme from U.S. Biochemical Corp. (Cleveland, Ohio) was used as directed by the supplier.

Synthetic complete medium (SCM) for yeast culture is $2 \%$ (wt/vol) glucose plus, per liter, $4.8 \mathrm{~g}$ of ammonium sulfate, 1.6 $\mathrm{g}$ of yeast nitrogen base without amino acids and without ammonium sulfate, $0.49 \mathrm{~g}$ of potassium phosphate (dibasic), 30 $\mathrm{mg}$ of Leu, $20 \mathrm{mg}$ each of Ade, Ura, His, Met, and Trp, $16.3 \mathrm{mg}$ each of Asp, Thr, and Val, $12 \mathrm{mg}$ of Phe, $7.3 \mathrm{mg}$ each of Ile, Lys, and Tyr, and $5 \mathrm{mg}$ each of Arg, Cys, Pro, and Ser. YPD medium for yeast culture is $2 \%$ glucose, $2 \%$ Bacto Peptone, and $1 \%$ Bacto Yeast Extract.

Plasmid preparation. Plasmids were transformed to $E$. coli by either electroporation or by the method of Hanahan as described elsewhere (38). Plasmids were isolated by alkaline lysis, and large-scale preparations of plasmids were further purified by step gradient sedimentation in cesium chloride (38).

Construction of linker mutations. Plasmid pARS/WT is a pUC119 derivative and has been described previously (27). It contains an ampicillin resistance gene, together with $U R A 3$ and CEN4 sequences. The $A R S 307$ fragment, R200, was excised from the R-200 pVHA plasmid (33) and cloned into the pARS/WT vector between HindIII and EcoRI sites. Linker substitutions were made as follows. Two sets of deletions from either end of the R200 fragment were generated. pARS/WT was first linearized with either EcoRI or HindIII and then treated with exonuclease III. Samples were removed at various time points, then blunt ended with DNA polymerase I Klenow fragment, and recircularized following ligation of an 8-bp XhoI linker (5'-CCTCGAGG-3'). Ligation mixes were transformed to $E$. coli; plasmids from 500 colonies were sized by gel electrophoresis, and a subset was sequenced to determine the deletion endpoint. The endpoints of the two sets of deletions were compared, and pairs that overlapped in $A R S 307$ by seven to nine bases were recombined at the XhoI linker site and another appropriate site on the plasmid. Alternatively, some constructs were made by oligonucleotide-directed mutagenesis.

Oligonucleotide-directed mutagenesis. Oligonucleotide-directed mutagenesis was performed by the method of Kunkel (25). This technique was used to make all the single-point mutations and three-base substitutions and also to interchange elements between $A R S 1$ and $A R S 307$.

Plasmid stability assay. $\mathrm{CsCl}$ gradient-purified plasmids were transformed into strain SP1 by a standard lithium acetate method (38). The plasmid stability assay was done as previously described (27). Yeast transformants were grown in selective (SCM-Ura) medium, diluted into nonselective medium (YPD), and then grown for $30 \mathrm{~h}$. Cultures were diluted and plated onto SCM and SCM-Ura plates, and the percentage of yeast cells that retained the plasmid was determined.

Purification of ORC. ORC was purified from $400 \mathrm{~g}$ of $S$. cerevisiae BJ926 whole-cell extract as described previously (2). The fractions containing ORC activity after passage through the ACS affinity column were used in the footprinting studies.

\section{RESULTS}

Mapping $A R S 307$ functional elements by linker scan mutagenesis. Linker scan analysis of $A R S 1$ revealed that it consists of one essential element, A, that contains a perfect match to the ACS and three important elements in the B domain, B1, B2, and B3 (27). While A-like elements are present in all ARSs and B3-like elements are found in a number of ARSs, attempts to identify B1- and B2-like elements by sequence similarity in other ARSs have failed.

To determine whether other ARSs have the same structural organization as $A R S 1$ or whether they have their own unique structure, we conducted a linker scan mutation analysis on another chromosomal replicator, $A R S 307$. The linker scan mutation technique was first successfully used to identify promoter elements that control gene expression (29). It is important to use this technique to study replicators because previous deletion studies on ARSs, including $A R S 1$ and $A R S 307(9,33)$, were inconclusive since the methods employed introduce large spacing changes. Deletion methods also did not detect functional redundancy, and they potentially suffer from interference by vector sequences. The linker scan technique worked well to define the structure of $A R S 1$, and the elements identified in the plasmid assay have been shown to be required for $A R S 1$ activity as a replicator in the chromosome (28).

ARS307 was cloned into the pUC119-derived wild-type vector (27). The R200 fragment contains $200 \mathrm{bp}$ and consists of overlapping 11-of-11 and 10-of-11 matches to the ACS and four 9-of-11 matches to the ACS $(33,34)$. On the basis of studies by Palzkill and Newlon (33), there seem to be no important sequences required for ARS function between nucleotides 1 and 77. Thus, we made 23 XhoI linker substitutions from positions 64 to 200 as shown in Fig. 1. In 15 of the mutants, 8 bp of the $A R S 307$ sequence were replaced with an 8-bp XhoI linker; in the other 8 mutants, either a 7- or 9-bp 


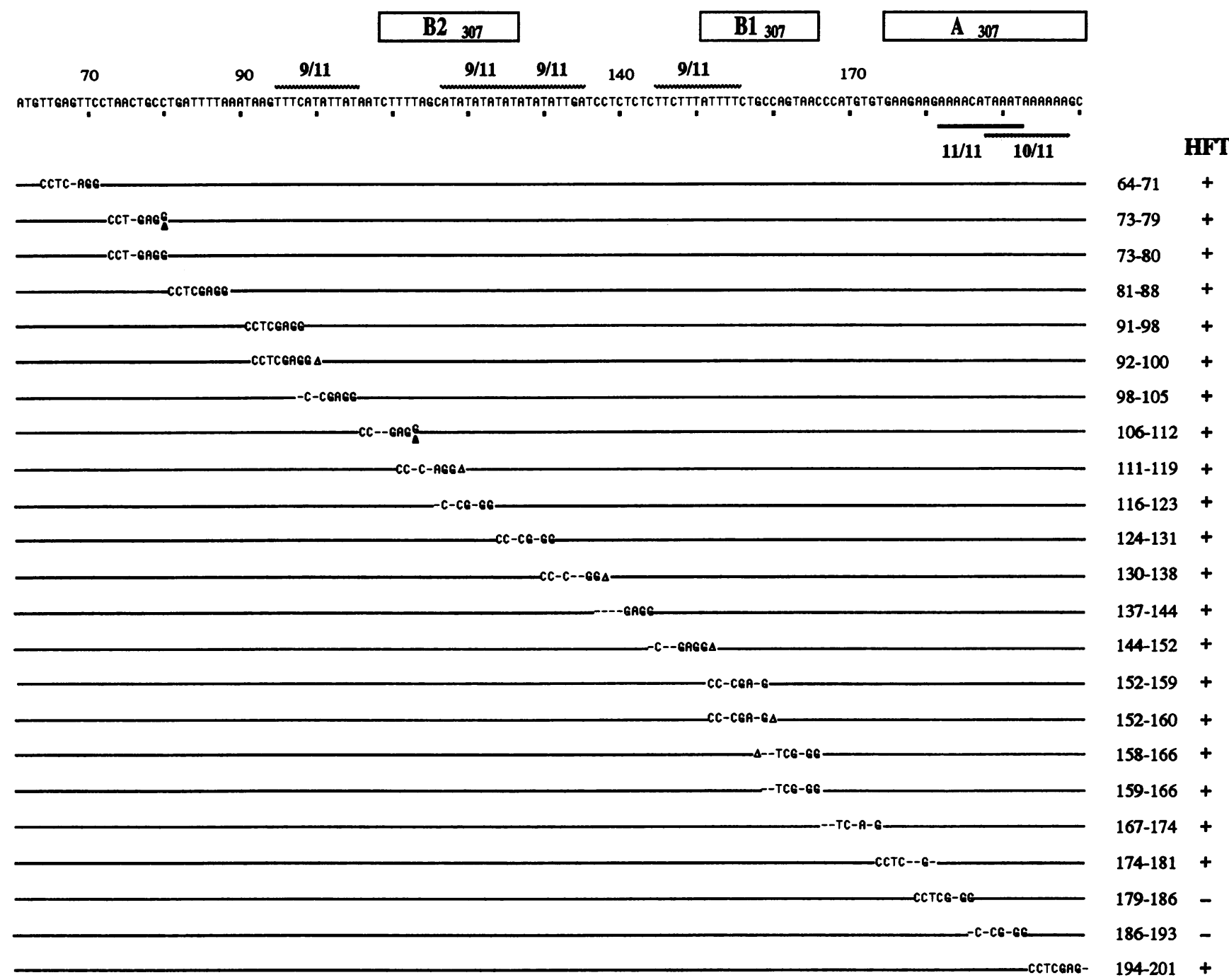

FIG. 1. Linker substitution mutations in ARS307. The wild-type ARS307 sequence (top) derives from the sequence initially characterized by Palzkill and Newlon (33). Each line below the wild-type sequence represents an XhoI linker substitution mutant. The sequence of each mutant is shown only at positions that differ from the wild-type $A R S 307$ sequence. The nucleotide positions of $X h o I$ linkers for each mutant are indicated at the immediate right. Most mutations contain an exact replacement of $8 \mathrm{bp}$; those that contain a 1-bp insertion or deletion are indicated with an arrowhead. All constructs contain the URA3 selectable marker and a CEN4 centromere. The results of the HFT test (27) are shown at the far right. A plus sign means the plasmid produces transformants at high frequency. The functional elements (A, B1, and B2) defined by the results described herein (Fig. 2 and 4 ) are marked by the boxes above the wild-type sequence. The perfect match (11 of 11) to the ACS is marked by the solid line. Other partial matches ( 9 of 11 and 10 of 11) are marked by the stippled lines.

sequence from $A R S 1$ was replaced with the XhoI linker (arrowheads in Fig. 1).

The plasmids, which contain a $U R A 3$ selectable marker and a centromere (CEN4), were transformed into yeast strain SP1 containing the mutant ura3-52 allele (27). Following transformation of SP1, plasmids carrying a functional replicator should produce URA $3^{+}$transformants. We found only two mutated plasmids which failed to produce transformants, and both of these mutants disrupted the 11-of-11 match to the ACS which had previously been shown to be essential $(8,10,20,27,28,33$, $36,44)$ (Fig. 1). In addition, we noticed slowly growing colonies when some of the plasmids were transformed and the yeast cells were selected on plates lacking uracil.

To further define the important but not individually essential sequences, all of the high-frequency transformation (HFT)positive plasmids were tested in a plasmid stability assay. The assay measures the percentage of yeast cells that retain the plasmid after growth for approximately $30 \mathrm{~h}$ in nonselective medium. Since the circular plasmids contain a centromere and efficiently segregate during mitosis, any changes in the stability of mutant plasmids most likely reflect changes in replicator activity. With this assay, most plasmids were as stable as the wild-type plasmid (Fig. 2A and B). However three groups of linker mutants showed reduced plasmid stabilities. The first group included two linker mutations in sequences flanking the 5' and 3' ends of the ACS; mutations 174-181 and 194-201 both have very low stabilities. Because of their very low plasmid stabilities and the fact that they lie adjacent to the ACS, we have included these mutations when defining the limits of the ARS307 A element. Thus, the sequence from nucleotides 174 to 200 is henceforth called $A_{307}$. In addition, we detected two clusters of linker scan mutations with reduced plasmid stabil- 
A

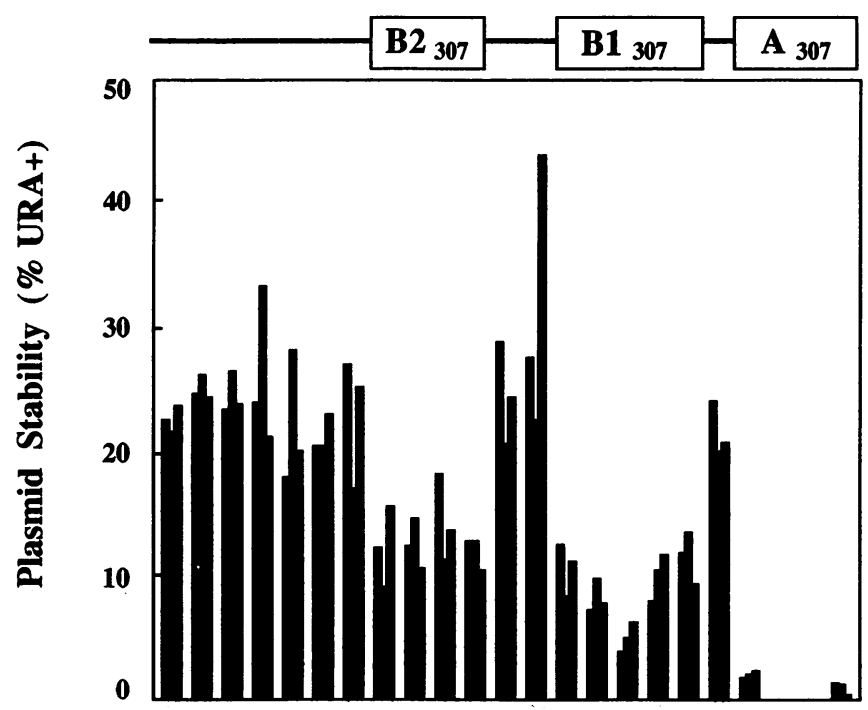

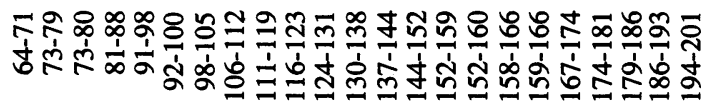

B

C

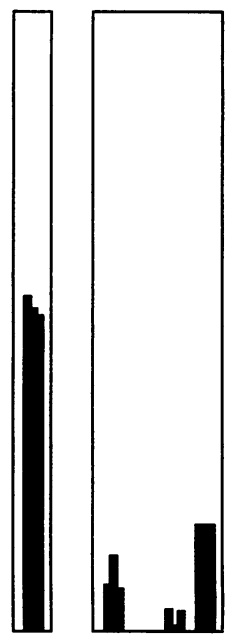

5

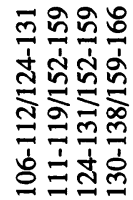

FIG. 2. Bar graphs of the plasmid stabilities of the wild-type $A R S 307$ (WT; B) and linker substitution mutants (A) shown in Fig. 1 and of double linker scan mutants and B1, B2 double mutants (C). The plasmid stability assay was done as described previously (27). The three bars for each plasmid construct represent data from three independent determinations. The functional elements defined by this assay are indicated at the top.

ities. Linker scan mutations defined the limits of two regions, from nucleotides 106 to 129 and 145 to 166 , that when altered reduced but did not eliminate plasmid stability. These two regions approximately correspond in position to the $\mathrm{B} 1$ and $\mathrm{B} 2$ elements in $A R S 1$; however, they have little sequence similarity with either the B1, B2, or B3 elements present in $A R S 1$.

To investigate further the importance of these two clusters in domain B, several double linker substitutions were made as shown in Fig. 2C. Plasmid 106-112/124-131 is a double linker mutation containing two linkers from the same cluster. This double mutant has a lower (but still measurable) plasmid stability than any single linker substitution located in that region. In contrast, plasmids $111-119 / 152-159$ and $124-131 / 152-$ 159 , which simultaneously have a linker scan mutation in each of the clusters, formed microcolonies on SCM-Ura plates, and the plasmid stabilities were extremely low or not measurable. To show that these severe effects were not simply caused by the introduction into the $A R S 307$ region of a GC-rich, 16-bp double linker mutation, we constructed plasmid 130-138/159166, which combined one mutation that reduced ARS function and another mutation that did not affect ARS function. This plasmid had the same stability as the single linker mutation which reduced ARS function (Fig. 2). These results show that two elements within the B region of $A R S 307$ are very important for replicator function. Since the spacing relative to the essential A element of these two clusters of mutations is similar to the location of the $A R S 1 \mathrm{~B} 1$ and $\mathrm{B} 2$ elements, we henceforth refer to them as $\mathrm{B} 1_{307}$ and $\mathrm{B} 2_{307}$, respectively.

ORC footprint on $A R S 307$ wild type and mutants. The multisubunit ORC was identified by the DNase I footprint method and binds to the ACS in $A R S 1$ and to the ACSs in all other ARSs that have been tested $(2,3)$. Mutations in the
ARS1 ACS that reduce or abolish the replicator activity also reduce or abolish ORC protection. Mutations in the ARS1 B elements had no effect on the DNase-I-induced ORC footprint. At high concentrations of ORC, or with mutations that abolish ARS1 A-element activity, ORC protection was observed over the $A R S 1 \mathrm{~B} 2$ element (3). The $\mathrm{B} 2$ element contains one 9-of-11 match to the ACS in the opposite orientation to the ACS found in the $A R S 1 \mathrm{~A}$ element. It was suggested that B2 might be an alternative site for ORC binding.

Similar footprint studies have not been done with mutants of other ARSs. To test specific binding by the putative initiator protein ORC, we performed footprinting studies on both wild-type and mutant $A R S 307$ plasmids with the affinity column fraction containing ORC activity (Fig. 3). ORC protects a 50-bp region, including the $\mathrm{A}$ element and part of the $\mathrm{B} 1$ element in $A R S 307$ (Fig. 3). Similar to that found at $A R S 1$, hypersensitive sites were seen at 10-bp intervals $3^{\prime}$ to the ACS (3). It has been suggested that these hypersensitive sites indicate that the DNA wraps around ORC. Two linker mutations in $\mathrm{A}_{307}, 179-186$ and 186-193, which abolish ARS activity, completely abolish the ORC footprint pattern on $A R S 307$. Unlike the case for $A R S 1$, there were no protections observed over any $A R S 307 \mathrm{~B}$ element when the essential A element was disrupted. Two linker mutations surrounding the ACS, 174-181 and 194-201, which greatly reduce ARS activity in the plasmid stability assay, also reduced the ORC footprint. However, the hypersensitive sites in domain B still remained. In contrast, mutations of $B 1_{307}$ plus $B 2_{307}$, which was shown to have greatly reduced plasmid stability, had no effect on the ORC footprint. These results indicate that the region from positions 174 to 201 containing the A element for $A R S 307$ also contains a binding site for ORC. 


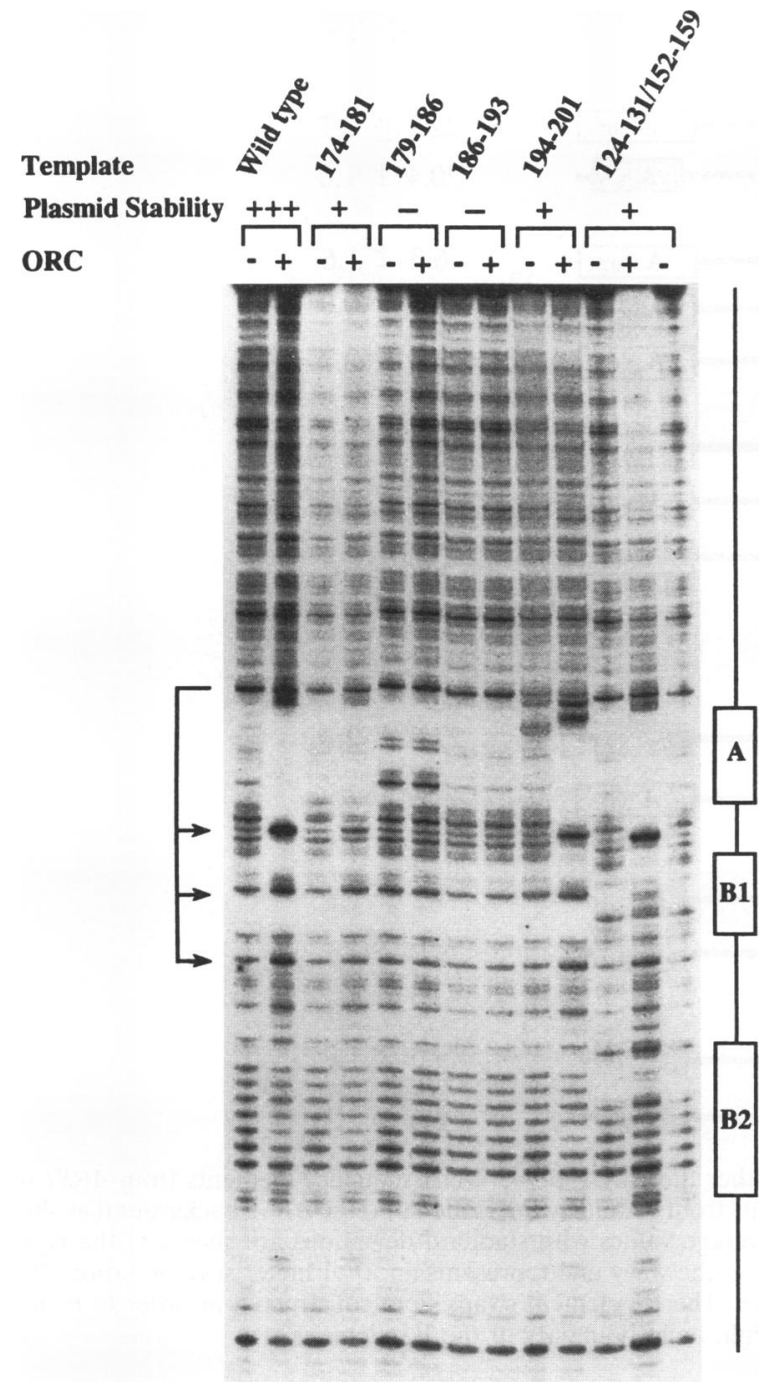

FIG. 3. DNase I footprint analysis of wild-type and mutant $A R S 307$ sequences with the yeast ORC. The ORC was purified as described by Bell et al. (2). A DNA affinity column fraction was used in this experiment. Wild-type or mutant $A R S 307$ DNA fragments labeled at position 1 and extending to position 522 were used for DNase I footprinting (33). The strand containing the A-rich sequence of the ACS match was labeled. Templates used are shown at the top. Plasmid stability:,$+++ 25 \% \mathrm{URA}^{+} ;+$, HFT positive, very low plasmid stability ( 0 to $5 \%)$; - , failed in HFT. The lanes that contain no protein are indicated as -; lanes that contain ORC $(120 \mathrm{ng})$ are indicated as + . The genetically defined DNA sequence elements in ARS307 are indicated at the right. The hypersensitive sites (arrows) and the region of protection (brackets) are shown at the left.

Mapping two regions in the $\mathrm{B}$ domain of $A R S 307$ by 3-bp substitutions. The two regions in the ARS307 domain B that were defined by linker scan analysis with an 8-bp linker corresponded in location to the $A R S 1 \mathrm{~B} 1$ and $\mathrm{B} 2$ domains. To define the $A R S 307$ B elements more precisely, 3-bp linker insertions were constructed and assayed for plasmid stability (Fig. 4). Since the ARS307 domain B is AT rich, the mutations were made by replacing three bases with a GGG trinucleotide except when the original sequence contained a $\mathrm{G}$, in which case it was changed to a $\mathrm{C}$.

To quantitatively assay the effects of these mutations, we

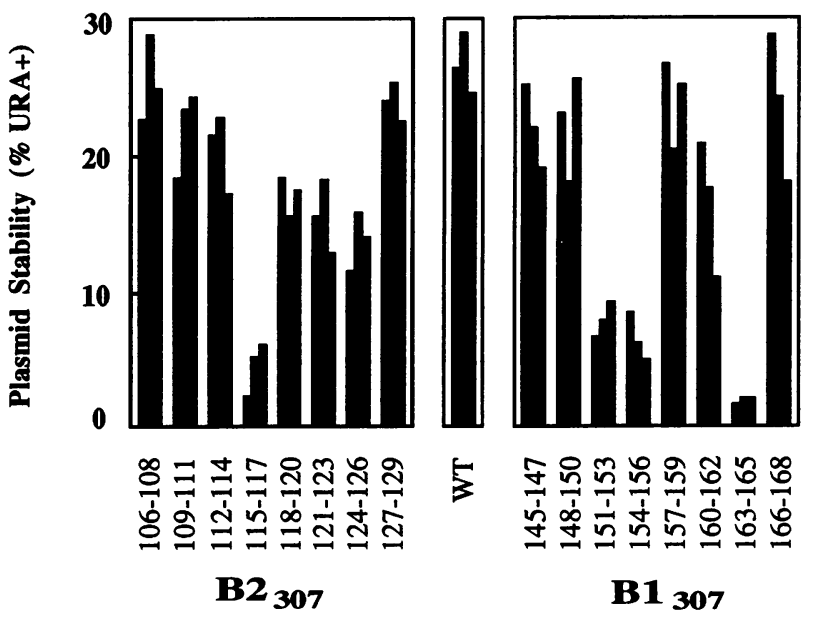

FIG. 4. Three-base-pair linker substitution mutations map the two B elements of $A R S 307$. The two $\mathrm{B}$ elements, $\mathrm{B} 1_{307}$ or $\mathrm{B} 2_{307}$, defined by 8-bp linker mutations were substituted by GGG linker substitutions. The horizontal axis shows the mutants by the 3-bp substitution coordinates in the two B elements. WT, wild-type ARS307.

again transformed the plasmids into strain SP1. The locations of the mutations and measurements in triplicate of the plasmid stabilities of these constructs are shown in Fig. 4. Within the $A R S 307$ B2 region, the most deleterious mutation was the 115-117 linker insertion, although three linker insertions from nucleotides 118 to 126 were reproducibly less stable than the wild-type plasmid. Although the 8-bp linker substitutions defined the maximal boundaries of the $\mathrm{B} 2$ region as mapping from nucleotides 106 to 129, the 3-bp linker insertions did not have a significant effect on $A R S 307$ function when inserted within nucleotides 106 to 114 and nucleotides 127 to 129 . This difference between the 8- and 3-bp linker insertions in the region from positions 106 to 114 might reflect some structural redundancy within the $\mathrm{B} 2$ element.

Three-base insertions in the ARS307 B1 defined two essential subdomains of the $\mathrm{B} 1$ region, located from nucleotides 151 to 156 and 163 to 165 . Mutation of the intervening region from nucleotides 160 to 162 only had a small effect on plasmid stability. Thus, these 3-bp linker scan mutations define in more detail the $A R S 307 \mathrm{~B} 1$ element. On the basis of the data from the 8- and 3-bp linkers, we defined the $A R S 307 \mathrm{~B} 2$ element as nucleotides 109 to 126 and the B1 element as nucleotides 151 to 165 .

$B 1$ and $B 2$ are distinct elements and are functionally conserved in yeast replicators. To address whether the elements identified in $A R S 307$ could functionally substitute for the B-region DNA sequence elements in $A R S 1$, the elements $\mathrm{A}_{1}, \mathrm{~B} 1_{1}$, and $\mathrm{B} 2_{1}$ from $A R S 1$ (27) were cloned in place of the various sequence elements in an $A R S 307$ background, using oligonucleotide mutagenesis (Fig. 5). $\mathrm{A}_{1}$ was positioned in $A R S 307$ so that the ACS was aligned in the same orientation as the original ACS. This chimeric plasmid had approximately the same plasmid stability as wild-type $A R S 307$. The reciprocal experiment, replacing the ARS1 A element $\left(\mathrm{A}_{1}\right)$ with the ARS307 A element $\left(\mathrm{A}_{307}\right)$, yielded similar results. This finding confirms that nucleotides 174 to 201 contain the A element in ARS307 and that the A elements are interchangeable between two ARSs.

The B elements were also swapped between the two ARS constructs. When the distal B element in $A R S 307\left(\mathrm{~B}_{307}\right)$ was replaced by $\mathrm{B} 1_{1}$ from $A R S 1$, the fusion plasmid had a very low 
A ARS1 Elements In ARS307

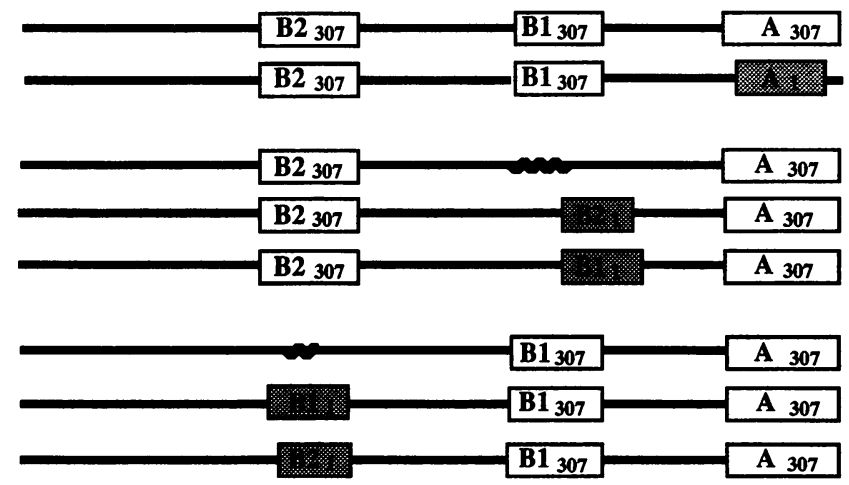

B ARS307 Elements In ARS1
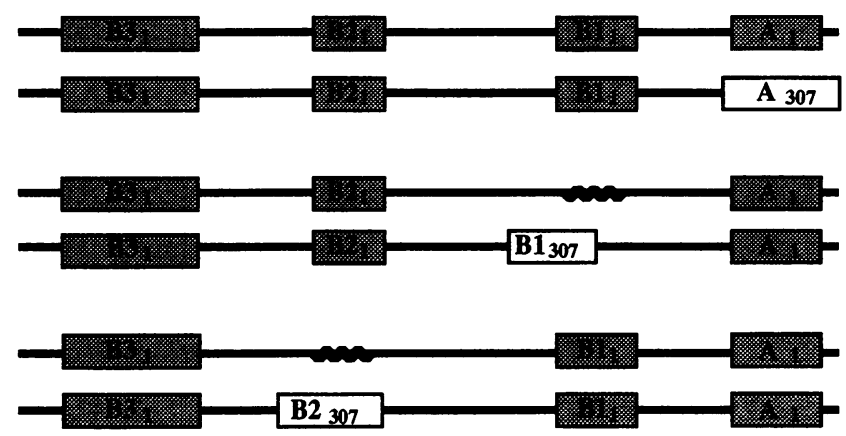

STABILITY (\%)

$20.4 \pm 1.0$

$6.3 \pm 1.6$

$0.4 \pm 0.0$

$27.7 \pm 2.7$

$4.0 \pm 1.1$

$7.0 \pm 1.2$

$22.5 \pm 2.4$
$27.8 \pm 2.8$

$31.7 \pm 2.0$

$4.2 \pm 1.3$

$26.7 \pm 1.1$

$5.0 \pm 1.9$

$12.6 \pm 2.2$

FIG. 5. Functional identity between the $A R S 1$ and $A R S 307$ elements. (A) Either the A, B1, or B2 DNA sequence elements from $A R S 1$ were cloned into the $A R S 307$ background as shown. (B) Either the A, B1, or B2 elements from $A R S 307$ were cloned into the $A R S 1$ background as shown. The plasmid stability of each construct was determined in triplicate, and the average values with standard deviations are shown at the right as percent URA ${ }^{+}$. The solid horizontal line represents wild-type DNA sequence, and the wavy line represents an XhoI linker scan mutation. White boxes represent $A R S 307$ sequences, and shaded boxes represent $A R S 1$ sequences. The B1 element swaps were constructed in order to maintain the spacing found in the corresponding ARS between the A element and the right-hand boundary of the B1 element.

plasmid stability, indicating that a B1 element could not substitute for a B2 element (Fig. 5). This finding is consistent with previous data for $A R S 1$ demonstrating that the $\mathrm{B} 1$ element could not substitute for the B2 element (27). Interestingly, when the $\mathrm{B} 2_{307}$ element in $A R S 307$ was replaced by $\mathrm{B} 2$, the chimera showed the same plasmid stability as wild-type $A R S 307$. Thus, the $\mathrm{B} 2_{307}$ element is functionally equivalent to the $\mathrm{B} 2$ element of $A R S 1$. Experiments of the same type were done with the proximal B element in $A R S 307, \mathrm{~B} 1_{307}$. This element could be interchanged with the $\mathrm{B} 1_{1}$ element from $A R S 1$ but not with the $A R S 1 \mathrm{~B} 2{ }_{1}$ element (Fig. 5). These results demonstrate that $\mathrm{B} 1$ and $\mathrm{B} 2$ are distinct elements and both are conserved between the two ARSs.

Analysis of three-base substitution mutations in $\mathrm{B}_{307}$ (Fig. 3) seemed to indicate that $\mathrm{B} 1_{307}$ has a bipartite structure. This raised the possibility that the full $\mathrm{B} 1_{307}$ element (151-165) might contain two elements. To test this, we replaced $\mathrm{B} 1_{1}$ from ARS1 with either the $160-165$ subelement of $\mathrm{B}_{307}$ or the full-length $\mathrm{B} 1_{307}(151-165)$. The 160-165 subelement could only partially substitute for $\mathrm{B} 1_{1}$ (data not shown). Only when the full-length $\mathrm{B} 1_{307}(151-165)$ was placed at the $\mathrm{B} 1_{1}$ position was wild-type $A R S 1$ activity recovered (Fig. 5). The $\mathrm{B}_{307}$ element, located at nucleotides 151 to 165 in $A R S 307$, may contain two subelements, but neither alone is sufficient for B1 function.
Effects of point mutations in the $B 1$ and $B 2$ elements of ARS1. To identify the nucleotide sequences important for $\mathrm{B} 1$ and $\mathrm{B} 2$ function, we made all possible (except one) single-base mutations in the core region of either the $\mathrm{B} 1$ or $\mathrm{B} 2$ elements in ARS1. The mutated core region of $\mathrm{B} 1$ is from nucleotides 837 to 842 (5'-AAAAGC-3'; Fig. 6). Two adenosine nucleotides at 838 and 839 appeared to be important for ARS activity, since

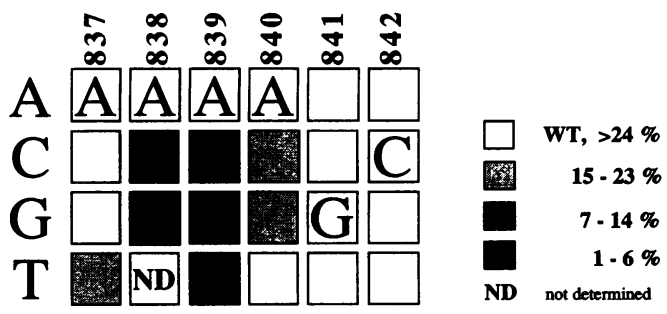

FIG. 6. Effects of point mutations in the B1 element of ARS1. The B1 element core sequence from $A R S 1$ nucleotides 837 to 842 is shown in each box. The wild-type (WT) sequence is 5'-AAAAGC-3'. Each base was mutated to all possible nucleotides except the $A$ to $T$ at position 838, which is shown as ND. The average results of three independent plasmid stability determinations are represented by boxes with different shades. 
single-nucleotide changes $(A-838 \rightarrow G$ and $A-839 \rightarrow$ C) reduced plasmid stability to the same level as a full 8-bp linker mutation (Fig. 6 and reference 27). Other mutations at these two positions were also much less stable than the wild type. Mutations in the base pairs adjacent to these two adenosine residues showed reduced plasmid stabilities. In contrast to these results with the $\mathrm{B} 1_{1}$ element, introduction of single-point mutations into the $\mathrm{B} 2{ }_{1}$ element from $A R S 1$ from nucleotides 801 to $806\left(5^{\prime}\right.$-TAAGTA-3') had no effect on the plasmid stability (data not shown).

\section{DISCUSSION}

The initiation of eukaryotic DNA replication is regulated, in part, by events that occur at the origin of DNA replication. These events are most likely controlled by interaction between an initiator protein and the DNA sequences that form the genetically defined replicator. A subset of the ARS sequences that promote initiation of DNA replication in a plasmid context also promote initiation of DNA replication in the chromosome and are thus chromosomal replicators. Analyses of ARS sequences both in a plasmid context $(4,5,8,9,18,20$, $27,33,44-46)$ and in the chromosome $(10,17,20,21,28,36)$ have led to the definition of replicators as multicomponent DNA sequence elements. In general, replicator sequences in $S$. cerevisiae consist of an essential A element that contains an exact or close match to a degenerate 11-bp ACS $(6,8,23,41)$ and adjacent flanking sequences. The ACS is essential for ARS activity in plasmids and for replicator function in the chromosome $(10,28,36,44)$, but it is clear that the ACS is not sufficient for replicator function $(4,9,20,27,28,33,45)$.

Certain DNA sequences that lie adjacent to the A element, $3^{\prime}$ to the T-rich strand of the ACS in the A element, are also essential for replicator function $(8,9,20,27,28,33)$. These sequences in the B domain of ARSs do not necessarily need to be derived from the yeast genome. For example, easily unwound DNA sequences can function in cooperation with the $A$ element for ARS activity $(20,32)$. Thus, the DNA sequence requirements in the $B$ domain for replicator function have remained elusive.

Because deletion mutagenesis has sometimes yielded contradictory results (4), we have focused on understanding replicator structure and function by systematically altering DNA sequences by using linker scan or point mutagenesis (27). Such an analysis does not dramatically alter the relative spacing between DNA sequences that may be important for initiation of DNA replication. Analysis of the ARS1 replicator from chromosome IV of $S$. cerevisiae has demonstrated that ARS1 contains an essential A element and three B elements, $\mathrm{B} 1, \mathrm{~B} 2$, and B3 (27). Recently we have demonstrated that these elements are also essential for $A R S 1$ function in the chromosomal context (28). In both the plasmid and chromosome contexts, mutation in any one of the B elements has a small effect on the replicator activity, but mutation of any two of the three B elements has a greater effect, and elimination of all three B elements causes the replicator to be inactive.

The results described in this report demonstrate the generality of this unusual modular nature of $S$. cerevisiae replicator sequences. $A R S 307$, a chromosomal replicator originally defined by Newlon and colleagues $(10,33,44)$, contains easily unwound DNA sequences adjacent to the essential A elements $(31,32)$. Analyses of linker scan mutations have confirmed that the A element containing the ACS is essential for replicator function $(10,44)$ and have identified two $B$ elements that correspond to the $\mathrm{B} 1$ and $\mathrm{B} 2$ elements found in $A R S 1$. Theis and Newlon (42) have performed a similar linker scan analysis of $A R S 307$ and have further demonstrated that the $A R S 307 \mathrm{~B} 1$ and $\mathrm{B} 2$ elements are required for initiation of DNA replication in the chromosome, just as the $A R S 1 \mathrm{~B} 1$ and $\mathrm{B} 2$ elements function in the chromosome (28). Previous deletion analyses have suggested that a number of close matches (9 of 11) to the ACS present in the B domain might be important ARS307 activity (33), but our analysis does not support this conclusion. Three-base linker scan mutations in both the $\mathrm{B} 2_{307}$ and $\mathrm{B} 1_{307}$ elements greatly reduce replicator function but do not alter the distribution of 9-of-11 matches to the ACS in ARS307 (Fig. 1 and 4). Rather, the $\mathrm{B} 1_{307}$ and $\mathrm{B} 2_{307}$ elements that we have defined are heretofore undetected DNA elements in ARS307 that are located in positions similar to those of the B1 and B2 elements found in $A R S 1$. Furthermore, the new $A R S 307$ elements can substitute for the $A R S 1 \mathrm{~B} 1_{2}$ and $\mathrm{B} 2{ }_{1}$ elements and thus probably perform similar functions.

The A elements in $A R S 1$ and $A R S 307$ have sequence similarity, as do all of the essential A elements in $S$. cerevisiae ARSs. It is thus not surprising that they can substitute for each other. In contrast, there is very little sequence similarity between the $A R S 1$ and $A R S 307 \mathrm{~B} 1$ or $\mathrm{B} 2$ elements, but surprisingly they can substitute for each other. A point mutation analysis of the $\mathrm{B} 1_{1}$ element from $A R S 1$ demonstrated that single-point mutations can cause a phenotype that is very similar to that caused by an 8-bp linker scan mutation in the $\mathrm{B} 1_{1}$ element. The two essential A nucleotides in the ARS1 B1 element are located 18 and 19 bases away from the end of the ACS. In $A R S 307$, two A nucleotides are located 17 and 18 bases away from the end of the ACS, and the 3-bp (163 to 165) linker scan that mutates these two A residues eliminates $B 1_{307}$ function. Thus, the B1 element, despite a lack of overall sequence similarity, seems to contain conserved nucleotides that are important. We suggest that the B1 element is the target for a protein(s) that could interact with these sequences, perhaps in cooperation with the ORC that interacts with the A and $\mathrm{B} 1$ regions of the replicators.

Single-point mutations in the core of the $A R S 1 \mathrm{~B} 22_{1}$ element did not show a phenotype, whereas 8-bp linker scan mutations in the $\mathrm{B} 2$ element from $A R S 1$ and $A R S 307$ and 3-bp mutations in the $\mathrm{B} 2_{307}$ element affected replicator activity. A linker scan mutation in the $\mathrm{B} 2$ element from $A R S 1$ in its natural location on chromosome IV has the most dramatic effect on origin activity of any of the single $B$ element mutations in that replicator (28). This observation might suggest that the B2 element plays a structural role, perhaps as a DNA-unwinding element $(20,31,32,43)$, or binds a protein with loose sequence specificity.

If the modular structure of $S$. cerevisiae replicators, with DNA elements that are conserved in function but not necessarily in sequence, is indicative of the nature of replicators in other eukaryotes, then these other replicators will be difficult to find and characterize. Yeasts offer the ability to clone replicators into extrachromosomal plasmids and genetically define their structures. In contrast, this has proven to be difficult in cells from metazoan species. Perhaps the replicators in other eukaryotes can be identified via the initiator proteins that might be more conserved.

The analysis of the DNA sequence elements required for yeast replicator function focuses attention on the A and B elements as targets for proteins that might participate in and regulate the initiation of DNA replication. We have demonstrated that the A element is a target for binding ORC in an ATP-dependent, sequence-specific manner, and the available genetic evidence supports a role for ORC in initiation of DNA replication $(2,3,11,16,26,28,30)$. The $\mathrm{B} 3$ element is the binding site for the transcription and DNA replication factor 
ABF1 and functions to stimulate replicator activity $(11,14,27$, $35,37,45)$. Understanding the protein-DNA and proteinprotein interactions between ORC and other proteins that may bind to ORC and/or the B1 and B2 elements at yeast replicator sequences should lead to insight into the now elusive mechanism of initiation of chromosomal DNA replication.

\section{ACKNOWLEDGMENTS}

We thank J. Scott, S. P. Bell, P. D. Kaufman, T. Melendy, and other members in the laboratory for helpful discussions. We also thank $\mathrm{C}$. Newlon and $\mathrm{J}$. Theis for communicating unpublished data and for valuable discussions.

This research was supported by grant AI 20460 from the National Institutes of Health.

\section{REFERENCES}

1. Amati, B., and S. M. Gasser. 1990. Drosophila scaffold attached regions bind nuclear scaffolds and can function as ARS elements in both budding and fission yeasts. Mol. Cell. Biol. 10:5442-5454.

2. Bell, S. P., R. Kobayashi, and B. Stillman. 1993. Yeast origin recognition complex functions in transcription silencing and DNA replication. Science 262:1844-1870.

3. Bell, S. P., and B. Stillman. 1992. ATP-dependent recognition of eukaryotic origins of DNA replication by a multiprotein complex. Nature (London) 357:128-134.

4. Bouton, A. H., and M. M. Smith. 1986. Fine-structure analysis of the DNA sequence requirements for autonomous replication of Saccharomyces cerevisiae plasmids. Mol. Cell. Biol. 6:2354-2363.

5. Brewer, B. J., and W. L. Fangman. 1987. The localization of replication origins on ARS plasmids in S. cerevisiae. Cell 51:463471.

6. Broach, J., Y. Y. Li, J. Feldman, M. Jayaram, J. Abraham, K. A. Nasmyth, and J. B. Hicks. 1983. Localization and sequence analysis of yeast origins of replication. Cold Spring Harbor Symp. Quant. Biol. 47:1165-1173.

7. Buchman, A. R., W. J. Kimmerly, J. Rine, and R. Kornberg. 1988. Two DNA binding factors recognize specific sequences at silencers, upstream activating sequences, and telomeres in Saccharomyces cerevisiae. Mol. Cell. Biol. 8:210-225.

8. Campbell, J. L., and C. S. Newlon. 1991. Chromosomal DNA replication, p. 41-146. In J. R. Broach, J. R. Pringle, and E. W. Jones (ed.), The molecular and cellular biology of the yeast Saccharomyces: genome dynamics, protein synthesis, and energetics. Cold Spring Harbor Laboratory Press, Cold Spring Harbor, N.Y.

9. Celniker, S. E., K. Sweder, F. Spienc, J. E. Bailey, and J. L. Campbell. 1984. Deletion mutations affecting automonously replicating sequence ARS1 of Saccharomyces cerevisiae. Mol. Cell. Biol. 4:2455-2466.

10. Deshpande, A., and C. Newlon. 1992. The ARS consensus sequence is required for chromosomal origin function in Saccharomyces cerevisiae. Mol. Cell. Biol. 12:4305-4313.

11. Diffley, J. F. X., and J. H. Cocker. 1992. Protein-DNA interactions at a yeast replication origin. Nature (London) 357:169-172.

12. Diffley, J. F. X., and B. Stillman. 1988. Purification of a yeast protein that binds to origins of DNA replication and a transcriptional silencer. Proc. Natl. Acad. Sci. USA 85:2120-2124.

13. Dubey, D. D., L. R. Davis, S. A. Greenfeder, L. Y. Ong, J. Zhu, J. R. Broach, C. S. Newlon, and J. A. Huberman. 1991. Evidence suggesting that the $A R S$ elements associated with silencer of the yeast mating-type locus $H M L$ do not function as chromosomal origins of replication. Mol. Cell. Biol. 11:5346-5355.

14. Eisenberg, S., C. Civalier, and B.-K. Tye. 1988. Specific interaction between a Saccharomyces cerevisiae protein and a DNA element associated with certain autonomously replicating sequences. Proc. Natl. Acad. Sci. USA 85:743-746.

15. Estes, H., B. Robinson, and S. Eisenberg. 1992. At least three distinct proteins are necessary for the reconstitution of a specific multiprotein complex at a eukaryotic chromosomal origin of replication. Proc. Natl. Acad. Sci. USA 89:11156-11160.

16. Foss, M., F. J. McNally, P. Laurenson, and J. Rine. 1993. Origin recognition complex (ORC) in transcriptional silencing and DNA replication in S. cerevisiae. Science 262:1838-1844.

17. Greenfeder, S., and C. Newlon. 1992. A replication map of a 61-kb circular derivative of Saccharomyces cerevisiae chromosome III. Mol. Biol. Cell 12:999-1013.

18. Holmes, S. G., and M. Mitchell. 1989. Interaction of the $\mathrm{H} 4$ autonomously replicating sequence core consensus sequence and its 3'-flanking domain. Mol. Cell. Biol. 9:5464-5472.

19. Hsiao, C.-L., and J. Carbon. 1979. High-frequency transformation of yeast by plasmids containing the cloned yeast $A R G 4$ gene. Proc. Natl. Acad. Sci. USA 76:3829-3833.

20. Huang, R., and D. Kowalski. 1993. A DNA unwinding element and an ARS consensus comprise a replication origin with a yeast chromosome. EMBO J. 12:4521-4531.

21. Huberman, J. A., J. Zhu, L. R. Davis, and C. S. Newlon. 1988. Close association of a DNA replication origin and an ARS element on chromosome III of the yeast, Saccharomyces cerevisiae. Nucleic Acids Res. 16:6373-6384.

22. Jacob, F., S. Brenner, and F. Cuzin. 1963. On the regulation of DNA replication in bacteria. Cold Spring Harbor Symp. Quant. Biol. 28:329-348.

23. Kearsey, S. 1984. Structural requirements for the function of a yeast chromosomal replicator. Cell 37:299-307.

24. Kornberg, A., and T. A. Baker. 1991. DNA replication. W. H. Freeman \& Co., New York.

25. Kunkel, T. 1985. Rapid and efficient site-specific mutagenesis without phenotypic selection. Proc. Natl. Acad. Sci. USA 82:488492.

26. Li, J. J., and I. Herskowitz. 1993. Isolation of ORC6, a component of the yeast origin recognition complex, by a one-hybrid system. Science 262:18701874.

27. Marahrens, Y., and B. Stillman. 1992. A yeast chromosomal origin of DNA replication defined by multiple functional elements. Science 255:817-823.

28. Marahrens, Y., and B. Stillman. 1994. Replicator dominance in a eukaryotic chromosome. EMBO J. 13:3395-3400.

29. McKnight, S. L., and R. Kingsbury. 1982. Transcriptional control signals of a eukaryotic protein-coding gene. Science 217:316-324.

30. Micklem, G., A. Rowley, J. Harwood, K. Nasmyth, and J. F. X. Diffley. 1993. Yeast origin recognition complex is involved in DNA replication and transcriptional silencing. Nature (London) 366:8789.

31. Natale, D. A., A. E. Schubert, and D. Kowalski. 1992. DNA helical stability accounts for mutational defects in a yeast replication origin. Proc. Natl. Acad. Sci. USA 89:2654-2658.

32. Natale, D. A., R. M. Umek, and D. Kowalski. 1993. Ease of DNA unwinding is a conserved property of yeast replication origin. Nucleic Acids Res. 21:555-560.

33. Palzkill, T. G., and C. S. Newlon. 1988. A yeast replication origin consists of multiple copies of a small conserved sequence. Cell 53:441-450.

34. Palzkill, T. G., S. G. Oliver, and C. S. Newlon. 1986. DNA sequence analysis of ARS elements from chromosome III of Saccharomyces cerevisiae: identification of a new conserved sequence. Nucleic Acids Res. 14:6247-6264.

35. Rhode, P. R., K. S. Sweder, K. F. Oegema, and J. L. Campbell. 1989. The gene encoding ARS-binding factor 1 is essential for the viability of yeast. Genes Dev. 3:1926-1939.

36. Rivier, D., and J. Rine. 1992. An origin of DNA replication and a transcription silencer require a common element. Science 256: 659-663.

37. Rowley, A., S. J. Dowell, and J. F. X. Diffley. 1994. Recent developments in the initiation of chromosomal DNA replication: a complex picture emerges. Biochim. Biophys. Acta 1217:239256.

38. Sambrook, J., E. F. Fritsch, and T. Maniatis. 1989. Molecular cloning, 2nd ed. Cold Spring Harbor Laboratory Press, Cold Spring Harbor, N.Y.

39. Stillman, B. 1993. Replicator renaissance. Nature (London) 366: 506-507.

40. Stillman, B. 1994. Initiation of chromosomal DNA replication in eukaryotes. J. Biol. Chem. 269:7047-7050.

41. Stinchcomb, D. T., K. Struhl, and R. W. Davis. 1979. Isolation and 
characterization of a yeast chromosomal replicator. Nature (London) 282:39-43.

42. Theis, J. F., and C. S. Newlon. 1994. Domain B of ARS307 contains two functional elements and contributes to chromosomal replication origin function. Mol. Cell. Biol. 14:7652-7659.

43. Umek, R. M., and D. Kowalski. 1988. The ease of DNA unwinding as a determinant of initiation at yeast replication origins. Cell 52: $559-567$.

44. van Houten, J. V., and C. S. Newlon. 1990. Mutational analysis of the consensus sequence of a replication origin from yeast chromosome III. Mol. Cell. Biol. 10:3917-3925.

45. Walker, S. S., S. C. Francesconi, and S. Eisenberg. 1990. A DNA replication enhancer in Saccharomyces cerevisiae. Proc. Natl. Acad. Sci. USA 87:4665-4669.

46. Walker, S. S., A. K. Malik, and S. Eisenberg. 1991. Analysis of the interactions of functional domains of a nuclear origin of replication from Saccharomyces cerevisiae. Nucleic Acids Res. 19:62556262 . 